\title{
Anthropometric, Physiological and Performance Characteristics of Elite and Sub-elite Fencers
}

\author{
by \\ Charilaos Tsolakis $\mathcal{E}$ George Vagenas ${ }^{1}$
}

\begin{abstract}
The present study aimed at investigating the differences in selected anthropometric, strength-power parameters and functional characteristics of fencing performance between elite and sub-elite fencers. Thirty-three fencers (18 females and 15 males) from the Greek National Team, (age $19 \pm 3.5 \mathrm{yr}$, body height $175.6 \pm 7.6 \mathrm{~cm}$, body mass 66.1 $\pm 9.1 \mathrm{~kg}$, systematic training $8.4 \pm 2.9 \mathrm{yr}$ ) were classified as elite and sub-elite, according to their international experience. Subjects underwent a detailed anthropometric assessment and performed selected leg power and fencingspecific tests. Significant differences were observed between the two groups in sitting height, triceps, subscapular, and quadriceps dominant skinfold thickness, absolute and body mass-dependent expressions of leg functional power characteristics of fencing performance: "time of lunge" and time of the "shuttle test". Anthropometric traits, such as height, body mass, percent fat and limb length were not different among elite and sub-elite fencers. Although technical and tactical factors are good indicators of fencing success, the observed differences in functional fencing performance tests among different levels of fencers are useful for the design of effective talent development and trainingconditioning programs for competitive fencers.
\end{abstract}

Key words: fencing, physiology, anthropometry,

\section{Introduction}

A number of studies provide information regarding physical fitness and anthropometric characteristics in various sports. The findings in most of these studies indicate significant differences in term of anthropometric and selected physical tests (sprinting, agility, vertical jumping, aerobic power) between young athletes of different levels or elite and non-elite athletes of soccer (Janssens et al., 2004; Reilly et al., 2000), handball (Zapartidis et al., 2009), hockey (Elferink-Gemser et al., 2004), and volleyball (Gabet and Georgieff, 2007; Smith et al., 1992; Thissen-Milder and Mayhew, 1991). On the other hand, recent studies have shown no significant difference in vertical jump and velocity of movement in the contact game between highly skilled and less skilled rugby players (Gabbet, 2009), as well as between winners and defeated karate players in anthropometric data and strength and vertical jump height, although winners tended to be more powerful in bench press and squat exercises (Roschel et al., 2009).

The importance of assessing sport-specific skills, as well as selected anthropometric and physiological characteristics in different sports, is vital to understanding sport performance, since the impact of high anthropometric and physical fitness qualities does not always transfer to improve playing performance (Gabbet et al., 2007).

In that respect, studies have shown that performance of high-level athletes could not be differentiated from that of novices on the basis of different motor tasks. Zehr et al. (1997) reported that peak velocity and movement time of a ballistic elbow extension, performed at maximal speed in a sitting posture, by competitive karate athletes or novices were not sig-

I - University of Athens, Department of Physical Education and Sports Science 
nificantly different, although this movement is frequently used in this sport. In French boxing, Beraud (1995) reported peak velocities of a kicking movement, performed in an upright posture at maximal speed, were essentially the same between a population of international boxing experts and novices. On the other hand, more recent studies found differences in ball velocity between first and second division handball players (Bayios et al., 2007), and between selected and non-selected handball players in a national team training camp (Zapartidis et al., 2009); while Gabbet (2009) detected powerful differences in tackling ability between the best and the worst rugby league players.

In more technical and less powerful sports, such as fencing, the existing results are rather unclear. Fencing is an intermittent sport that requires the fencers to compete in frequent, short bouts of high intensity exercise, followed by periods of low intensity activity. Bounces, steps of different direction and lunges occur repeatedly during the competition for the purpose of hitting the opponent, which also puts considerable demands on the neuromuscular system (Czajkowski, 2005). The distinct combination of longitudinal dimensions, such as stature, arm span, leg length, flexibility, muscular strength, muscular power, and inter-limb coordination, are necessary for successful performance and influencing fencingspecific motor abilities (Barth and Beck, 2007).

Harmenberg et al. (1991) showed that in terms of reaction time and movement time of a lunge movement performed from the initial garde position, world class fencers could not be differentiated from beginners. However, when initiation of the lunge movement occurred while moving backwards (retreating), the movement time of the lunge was shorter in the skilled compared to the novice fencers.

The inter-group comparison of another fencing study (Yiou and Do, 2001) has reported that the speed performance of a fencing touch was about equal between two groups of different levels in the isolated condition, but faster in expert versus novice athletes in the touch-lunge condition. When EMG analysis is used to measure response timing and neuromuscular coordination of the fencing lunge, the onset of muscle activity was significantly faster in the elite group in five of six muscles studied, and elite fencers showed more coherent muscle synergies and more consistent patterns of muscle coordination compared to novice fencers (Williams and Walmsley, 2000).
The available data regarding motor abilities and specific anthropometric characteristics in elite and sub-elite fencers are rather inconclusive and sparse. Thus, the aim of the present study investigates selected characteristics of the anthropometric profile, the strength-power profile and the functional fencing performance in elite and sub-elite fencers. It was hypothesized that elite fencers will be superior to subelite fencers in these characteristics.

\section{Materials and Methods}

\section{Participants}

The sample consisted of 33 members of the Greek National (18 females and 15 males) fencing Team (age $19 \pm 3.5 \mathrm{yr}$, body height $175.6 \pm 7.6 \mathrm{~cm}$, body mass $66.1 \pm 9.1 \mathrm{~kg}$, systematic training $8.4 \pm 2.9 \mathrm{yr}$ ). Fourteen were classified as elite, having competed at the Olympic Games and the World Championships; while the remaining 19 were classified as sub-elite, having had adequate experience in international competitions. Prior to data collection and after a thorough description of the risks being involved in the procedural steps, informed consent was obtained from each subject. The study was approved by the Institutional Review board of the Department of Physical Education and Sports Science at the University of Athens.

\section{Study design}

Subjects were tested on two separate sessions with a two-day break. The first session involved detailed anthropometric assessments, while field tests were performed in the second session. Standard warm-up procedures were given with $10 \mathrm{~min}$ of jogging, dynamic stretching activities of the lower extremity and a couple of trials for each test. The subjects were fully familiar with the exercise testing procedures, since they routinely performed these tests for training regimen purposes. In the 24-hour period before performing the tests, the subjects did not engage in any fatiguing activity in regards to stretch shortening cycles (SSC) and specific fencing tests. The order of the jumps and fencing tests was systematically balanced to minimize carry over effects (fatigue and learning). Each player was verbally instructed and encouraged during each test to perform maximally at each trial. 


\section{Anthropometry}

The anthropometric parameters included height (Hgt), sitting height (SHgt), weight $(\mathrm{Wt})$, humerus and femur breadths, arm, forearm, thigh and calf girths, biceps, triceps, subscapular, suprailiac, midthigh and calf skinfolds. Leg length (LL) was estimated as height minus sitting height (SHgt) and arm span (AS) as the distance between open hands at the shoulder level. All measurements were taken twice on the dominant side (fencing arm) of each subject. The girths and skinfolds were obtained by measurements on both sides. The test-retest reliability of the measurements was estimated to be 0.98 , ( $p<$ $.001)$. Subjects' height and body mass were measured to the nearest $0.1 \mathrm{~cm}$ and $0.1 \mathrm{~kg}$, respectively. The girths were measured with a Lafayette Gullick anthropometric tape (Model J00305). Skin folds were taken using a Harpenden skin fold caliper and percent (\%) body fat was estimated according to Durnin and Rahaman (Durnin and Rahaman, 1967). Lean body mass (LBM) was calculated by subtracting fat mass from body mass. Body Mass Index (BMI) was calculated as body mass per (height) ${ }^{2}$ in $\mathrm{kg} / \mathrm{m}^{2}$. Midthigh cross-sectional areas (CSAs) were calculated by an anthropometric formula, incorporating limb circumference and skinfolds as proposed by Heymsfield et al. (1982).

\section{Testing procedures}

Subjects were tested on their standing reach height with their arms down at the side before they performed a squat jump (SJ), a countermovement jump (CMJ), an arm-driven countermovement jump (ACMJ) and a drop jump from $40 \mathrm{~cm}$ (DJ40).

Jumping performance was determined using an Ergojump contact platform (Ergojump, Psion XP, MA.GI.CA., Rome, Italy) as described by Bosco et al. (1983). All subjects were instructed to leave the mat with the knees and ankles extended and land in an upright position. The test-retest reliability for the SJ, CMJ, ACMJ and DJ tests was estimated to be 0.94, $0.98,0.97$ and 0.87 , respectively $(p<.001)$.

Two fencing-specific tests were given to the subjects: The "time of lunge" (TL) and the time of the "shuttle test" (ST) were recorded by means of four photocells (Polifermo radio Light - Microgate Italy). For the "time of lunge", the photocells were placed at an adjustable lunge distance (2/3 leg length) according to Yiou and Do (2000), and the participants were asked to execute a fencing lunge at maximal speed. The height of the photocells was adjusted to be interrupted by the chest of the athletes. For the "shuttle test", which included three separate tests, "5 $\mathrm{m}$ forward $-5 \mathrm{~m}$ backward with fencing steps", the photocells were placed at the start and at the end of the $5 \mathrm{~m}$ distance. The subject takes on the guard position behind the starting line and moves with correct fencing steps forward and back between two parallel lines as fast as possible to cover a total distance of $30 \mathrm{~m}$ (Iglesias and Rodrigez, 2008). Both fencing tests were performed with the subject wearing fencing shoes and the lower part of the fencing uniform, without holding any weapon. These tests were chosen on the basis of their relative simplicity and their close specificity to functional fencing performance, as well as the comfortable familiarization with the testing procedures. The test-retest reliability for the "time of lunge" and the "shuttle test" was estimated to be 0.93 and 0.98 , respectively $(p<.001)$.

In all tests, the better of two trials was recorded for further processing. All tests were performed with a 30s rest between trials, while the rest phase between two consecutive tests was approximately 5 min. Exception was the procedures of the shuttle test. A rest of 2 minutes between trials was included to minimize the effects of fatigue.

\section{Derived Indexes}

Reactive strength index (RSI), derived from the height achieved during the DJ divided by the ground contact time $(\mathrm{cm} / \mathrm{sec})$. RSI is used as an indicator of the ability of the athlete to overcome different stretch loads during SSC activities. This index is also referred to as leg stiffness index. (Young et al., 1995)

Elasticity index (EI) is an indirect way to determine reactive strength. The difference between CMJ and SJ heights measures muscles' ability to use the elastic components, which are activated during the eccentric phase of the CMJ. (Bosco et al., 1983; Young et al., 1995)

\section{Statistical analysis}

All variables were tested for their conformity to the assumption of distributional normality using proper histograms and Kollmogorov-Smirnoff tests. The results are presented as means and standard deviations. Differences between groups were tested by student's t-test for independent variables. Significance was set at the probability level of $\alpha=0.05$. 


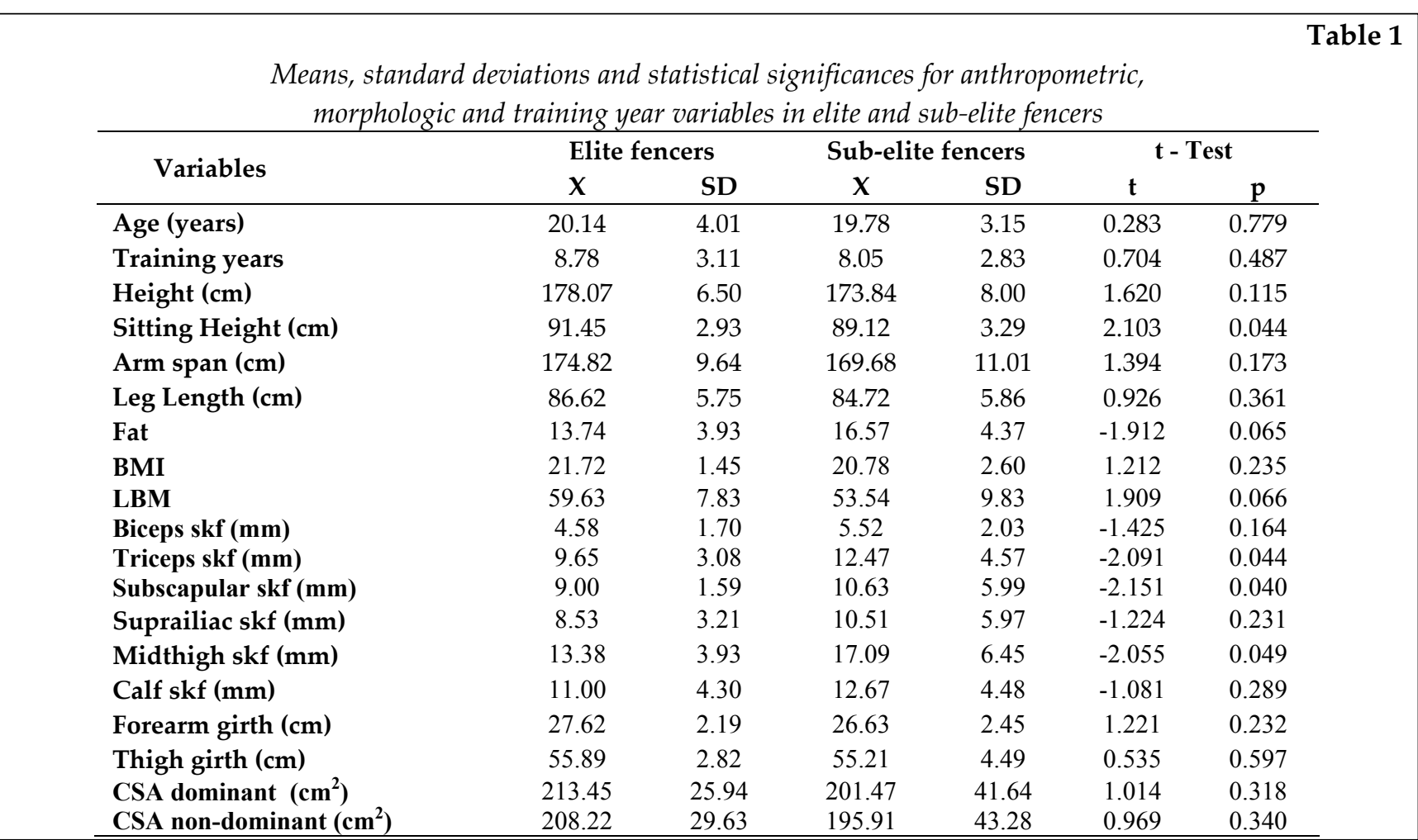

\section{Results}

Means and standard deviations of the anthropometric and morphological characteristics of the subjects are presented in Table 1, while means and standard deviations for the strength-power parameters and the functional characteristics of fencing performance are presented in Table 2. Statistically significant differences $(\mathrm{p}<0.05)$ were observed between elite and sub-elite fencers in sitting height $(\mathrm{p}=0.044)$, triceps ( $\mathrm{p}=0.044)$, subscapular $(\mathrm{p}=0.040)$, and quadriceps dominant $(\mathrm{p}=0.049)$ skinfold thickness, absolute and body mass dependent expressions of leg functional power characteristics of fencing performance: "time of lunge" $(\mathrm{p}=0.041-0.007)$ and the time of the "shuttle test" ( $p=0.011-0.014)$, respectively. No significant differences were observed between the two groups for the non-trainable measures of the fencers (height, leg length and arm span), body composition measures (\%fat, BMI, wt) and strength-power parameters (SJ, CMG, DJ). Nevertheless, elite fencers had a greater height, leg length and arm span and achieved better results in the above mentioned strength-power parameters than sub-elite fencers.

\section{Discussion}

The present study is the first to investigate differences in anthropometric, strength-power parameters and power characteristics of leg functional performance between elite and sub-elite fencers. The results of this study demonstrate that significant differences exist in sitting height, triceps, subscapular, and quadriceps dominant skinfold thickness and in absolute and body mass dependent expressions of leg functional power characteristics of fencing performance ("time of lunge" and time of "shuttle test").

Previous studies have investigated anthropometric and physiological qualities of athletes competing at different playing levels (Elferink-Gemser et al., 2004; Gabet and Georgieff, 2007; Janssens et al., 2004; Reilly, et al., 2000; Zapartidis et al., 2009), while a few studies have investigated skill differences between elite and non-elite athletes (Bayios et al., 2007; Beraud, 1995; Gabbet, 2009; Zapartidis et al., 2009; Zehr et al., 1997). Thus, it appears that the present study provides some useful data regarding fencing at the elite and sub-elite level.

Lunge is arguably the most important skill in fencing, with fencing success partially dependant upon maximum strength and power of dominant and non-dominant leg muscles (Nystrom et al., 1990; Sapega, et al., 1978), joint angle characteristics and high speed performance (Adrian and Klinger, 1983; Yiou and Do, 2000), time to peak force (Cronin, et al., 2003), different lengths of execution and the muscular coordination between the lower limbs. Especially, during competition the game requires longer phases 
Table 2

Means, standard deviations and statistical significances for strength-power parameters and functional characteristics of fencing performance in elite and sub-elite fencers

\begin{tabular}{lcccccc}
\hline \multicolumn{1}{c}{ Variables } & \multicolumn{2}{c}{ Elite fencers } & \multicolumn{2}{c}{ Sub-elite fencers } & \multicolumn{2}{c}{$\mathbf{t}-$ Test } \\
& $\mathbf{X}$ & SD & $\mathbf{X}$ & SD & Sign. & $\mathbf{p}$ \\
\hline Squat jump (cm) & 31.94 & 9.79 & 25.74 & 6.43 & 2.063 & 0.052 \\
Counter jump (cm) & 35.47 & 8.97 & 31.04 & 7.63 & 1.461 & 0.157 \\
Elasticity & 3.53 & 6.43 & 5.30 & 3.63 & -0.926 & 0.366 \\
Drop jump (cm) & 34.26 & 9.95 & 286.10 & 67.68 & 1.835 & 0.080 \\
Contact time Drop jump (msec) & 243.71 & 72.72 & 219.57 & 57.01 & 1.030 & 0.313 \\
Arm Counter jump (cm) & 41.44 & 8.99 & 36.19 & 8.22 & 1.717 & 0.098 \\
Reaction Strength Index & 1.48 & 0.64 & 1.38 & 0.47 & 0.520 & 0.608 \\
Time of lunge (msec) & 0.18 & 0.03 & 0.21 & 0.04 & -2.138 & 0.041 \\
Time of lunge/WT (msec)/kg & 0.27 & 0.04 & 0.34 & 0.08 & -2.893 & 0.007 \\
Shuttle test (sec) & 12.43 & 0.95 & 13.28 & 0.93 & -2.699 & 0.011 \\
Shuttle test/WT (sec)/kg & 0.18 & 0.02 & 0.21 & 0.04 & -2.608 & 0.014 \\
\hline
\end{tabular}

of fencing steps, which may influence the kinetic patterns and the hit accuracy of the fencing lunge (Czajkowski, 2005). Given the importance of these factors and their potential association to fencing performance, the time of lunge and the shuttle test was expected to discriminate between our two groups of fencers. Indeed, elite fencers were faster in both tests, in comparison to their sub-elite counterparts. This finding may reflect the higher training and competition intensity at the elite level (Harmenberg et al., 1991; Yiou and Do, 2001). However, the scarcity of literature data concerning anthropometric and physical fitness characteristics of elite, compared to their sub-elite fencers, make the interpretation of the current results difficult.

Expert fencers are known to possess, during retreat, shorter movement times of the lunge compared to novice fencers (Harmenberg et al., 1991), and higher speed performance in the touch-lunge condition (Yiou and Do, 2001). This trend was also evident in EMG analysis, in which elite fencers possessed faster onsets of muscular activity with better muscle synergies and pattern of coordination (Wiiliams and Walmsley, 2000).

We hypothesized some differentiation on the basis of vertical jumps, via indexes of lower body muscular power (Markovic and Jaric, (2007). However, it appears that the association between leg power tests and functional fencing performance is yet unfounded, as evidence has not shown significant differences between good and poor tacklers in vertical jump height (Gabbet, 2009; Gabbet et al., 2009). On the other hand, the lack of significant differences in all four vertical jump tests in the present study may reflect the insufficiency of these power tests in the sensitivity required to detect differences among elite and sub-elite fencers.

Finally, the anthropometrics, as a whole, did not produce a significant differentiation between the elite and sub-elite groups of fencers, which indicates that perhaps the few exceptions regarding sitting height and lower triceps, subscapular, and quadriceps dominant skinfold thicknesses, are sample-specific results or due to random variation of these properties. A clear superiority of the elite fencers group was expected, given the critical role of body size in the functional aspect of fencing performance. It appears that the classical anthropometrics of height, body mass, percent body fat, limb length, and various body and segment circumferences are simple descriptors, rather than determinants of leg functional power characteristics of performance in fencing (Tsolakis et al., 2006). The lower skinfold thickness may be advantageous for faster segmental movements and lower physiological demands during fencing. Although some morphological factors of the athletes seem to be important in fencing success, their influence is rather small when physiological, technical and tactical factors are accounted for (Roi and Bianchedi, 2008).

\section{Conclusion}

The present study investigated differences in selected anthropometric characteristics, physical fitness and power characteristics of fencing-specific tests between elite and sub-elite fencers. The main research question was to establish which properties of anthropometry and fencing skills are sub-elite fencers inferior to the elite, as to improve sub-elite fencers to the elite level. The results are quite clear 
that elite fencers are superior in fencing-related functional tests compared to sub-elite fencers. With a few exceptions, elite fencers are very similar to subelite in most of the anthropometric traits, which may indicate that other factors determine the formation of the top two levels of fencing. Further studies are required to identify relationships between selected anthropometric, strength-power parameters and fencing-specific skills, in order to provide additional evidence regarding the incorporation of certain training methodologies into the design of effective talent-development programs in fencing.

\section{References}

Adrian M., Klinger A. A biomechanical analysis of the fencing lunge. Proceedings of the Eighth International Congress of Biomechanic, Human Kinetics, 1983; 882-888.

Barth B., Beck E. The complete guide to fencing. Oxford: Meyer and Meyer sport (UK) Ltd. 2007.

Bayios I.A., Anastasopoulou E.M., Sioudris D.S., Boudolos. Relationship between isokinetic strength of the internal and external shoulder rotators and ball velocity in team handball. J Sports Med Phys Fitness, 2007; 41: $229-235$.

Beraud P. Analyse experimentale des coordinates posturo-cinetique liees a la delivrance d'un coup de pied. Cas de boxe francaise savate. University Aix-Marseille II, PhD thesis. 1995.

Bosco C., Luhtanen P., Komi P.V. A simple method for measurement of mechanical power in jumping. Eur JAppl Physiol, 1983; 50: 273-282.

Cronin J., McNair P.J., Marshall R.N. Lunge performance and its determinants. J Sports Sci, 2003; $21: 49-57$.

Czajkowski Z. Understanding Fencing. SKA Swordplay Books. 2005.

Durnin J.V.G.A., Rahaman M.M. The assessment of the amount of fat in the human body from measurements of skinfold thickness. Br J Nutrition, 1967; 21: 681-689.

Elferink-Gemser M., Visscher C., Lemmink K., Mulder T. Relation between multidimensional performance characteristics and level of performance in talented youth field hockey players. J Sports Sci, 2004; 22: 1053-1063.

Gabbett T., Georgieff B. Physiological and anthropometric characteristics of Australian junior national state, and novice volleyball players. J Strength Cond Res, 2007; 21(3): 902-908.

Gabbett T., Kelly J., Ralph S., Driscoll D. Physiological and anthropometric characteristics of junior elite and subelite rugby league players, with special reference to starters and non-starters. J Sci Med Sport, 2009; 12: 215-222.

Gabett T.J. Physiological and anthropometric correlates of tackling ability in rugby league players. J Strength Cond Res, 2009; 23(2): 540-548.

Harmenberg J., Ceci R., Barvestad P., Hjerpe K. Nystrom J Comparison of different tests of fencing performance. International Journal of Sports Medicine, 1991; 12: 573-576.

Heymsfield S.B., MCManus C., Smith, J., Stevens, V., Nixon DW. Anthropometric measurement of muscle mass: revised equations for calculating bone-free arm muscle area. Am. J Clin Nutr, 1982; 36: 680-690.

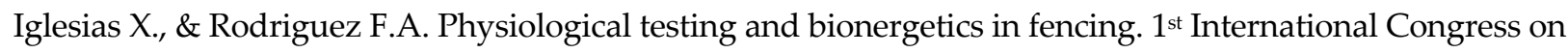
Science and Technology in Fencing. 15-17 February, Barcelona. Book of Abstract, 2008; 32-34.

Janssens M., Van Renterghem B., Bourgois J., Vrijens J. Physical fitness and specific motor performance of young soccer players aged 11-12 years. J Sports Sci, 2000; 16: 434-435.

Markovic G, Jaric S. Is vertical jump height a body size-independent measure of muscle power? J Sports Sci. 2007; 25 (12):1355-63.

Nystrom J., Lindwall, O., Ceci, R., Harmenberg, J., Swedenhag, J., Ekblom, B. Physiological and morphological characteristics of world class fencers. Int J Sports Med, 1990; 11(2): 136-139. 
Reilly T., Williams A., Nevill A., Franks A. A multidisciplinary approach to talent identification in soccer. J Sports Sci, 2000; 18: 695-702.

Roi G.S., Bianchedi D. The science of fencing: implications for performance and injury prevention. Sports Med, 2008; 38(6): 465-481.

Roschel H., Batista M, Monteiro R., Bertuzzi R.C., Barroso R., Loturco I., Ugrinowitsch C., Tricoli V., Franchini E. Association between neuromuscular tests and kumite performance on the Brazilian Karate national team. J Sports Sci Med, 2009; 8 (CSSI 3): 20-24.

Sapega A.A., Minkoff J., Nickolas J.A., Valsamis M. Sport specific performance factor profiling. Fencing as a prototype. Am J Sports Med, 1978; 6: 232-235.

Smith D.J., Roberts D., Watson B. Physical, physiological and performance differences between Canadian national team and universiade volleyball players. J Sports Sci. 1992; 10: 131-138.

Thissen-Milder, M., Mahew, J.L. Selection and classification of high school volleyball players from performance tests. J. Sport Med Phys Fitness, 1991; 31: 380-384.

Tsolakis C., Bogdanis G.C., Vagenas G. Anthropometric profile and limb asymmetries in young male and female fencers. J Hum Mov Stud, 2006; 50: 201-216.

Williams L.R.T., \& Walmsley A. Response timing and muscular coordination in fencing: a comparison of elite and novice fencers. J Sci Med Sports, 2000; 3(4): 460-475.

Yiou E., Do M.C. In fencing, does intensive practice equally improve the speed performance of the touché when it is performed alone and in combination with the lunge? Int J Sports Med, 2000; 21(12): 122-126.

Yiou E., Do M.C. In a complex sequential movement, what component of the motor program is improved with intense practice, sequence timing or ensemble motor learning? Exp Brain Res, 2001; 137: 197-204.

Young W., McLean B., Ardagna J. Relationship between strength qualities and sprinting performance. J Sports Med Phys Fitness, 1995; 53: 13-19.

Zapartidis I., Vareltzis I., Gouvali M., Kororos P. Physical fitness and anthropometric characteristics in different levels of young team handball players. The Open Sports Sciences Journal, 2009; 2: 22-28.

Zehr E.P., Sale D.G., Dowling J.J. Ballistic movement performance in karate athletes. Med Sci Sports Exerc, 1997; 29: 1366-1373.

\section{Corresponding author}

\section{Tsolakis Charilaos}

Assistant Prof. University of Athens

Department of Physical Education and Sports Science

6 Kotsika Street, 10434, Athens, GREECE,

Phone: ++30 210-727-6028

Fax: ++30 210-727-6028

E-mail. tsolakis@phed.uoa.gr 